PROCEEDINGS OF THE

AMERICAN MATHEMATICAL SOCIETY

Volume 135, Number 3, March 2007, Pages 837-844

S 0002-9939(06)08540-6

Article electronically published on August 31, 2006

\title{
NON-CUPPING AND RANDOMNESS
}

\author{
ANDRÉ NIES
}

(Communicated by Julia F. Knight)

\begin{abstract}
Let $Y \in \Delta_{2}^{0}$ be Martin-Löf-random. Then there is a promptly simple set $A$ such that for each Martin-Löf-random set $Z, Y \leq_{T} A \oplus Z \Rightarrow$ $Y \leq_{T} Z$. When $Y=\Omega$, one obtains a c.e. non-computable set $A$ which is not weakly Martin-Löf cuppable. That is, for any Martin-Löf-random set $Z$, if $\emptyset^{\prime} \leq_{T} A \oplus Z$, then $\emptyset^{\prime} \leq_{T} Z$.
\end{abstract}

\section{INTRODUCTION}

The interaction between $K$-triviality and Martin-Löf randomness via Turing reducibility is not very well understood at present. There are numerous results indicating a strong interaction, but as many open questions remain. Recall that a set $A \subseteq \mathbb{N}$ is $K$-trivial if, up to a constant, the initial segments of $A$ have minimum complexity, namely $(\forall n) K(A\lceil n) \leq K(n)+\mathcal{O}(1)$. (Here $K(x)$ is the prefix free complexity of $x$, and one identifies a string $\sigma$ in $2^{<\omega}$ with the natural number $n$ such that the binary representation of $n+1$ is $1 \sigma$.) This notion is opposite to MartinLöf-randomness (ML-randomness, for short), since $Z$ is ML-random iff there is a constant $b$ such that $(\forall n) K(Z\lceil n) \geq n-b$, namely the initial segments of $Z$ have close to maximal complexity. $K$-trivial sets have been studied for instance in [3, 11. An example of an interaction between $K$-triviality and Martin-Löf randomness via Turing reducibility is the following result from [4].

Theorem 1.1. Let $A$ be c.e. If $A \leq_{T} Z$ for some $M L$-random set $Z$ such that $\emptyset^{\prime} \mathbb{Z}_{T} Z$, then $A$ is $K$-trivial.

In [5], Kučera provides an injury-free solution of Post's Problem. To do so, given any $\Delta_{2}^{0}$ ML-random set $Z$ (and in fact any $\Delta_{2}^{0}$ diagonally non-computable set), he builds a non-computable c.e. set $A \leq_{T} Z$. Then, since there is a low ML-random set, a non-computable set $A$ satisfying the hypotheses of the theorem exists. It is an open question whether each $K$-trivial set is of this kind [8, Question 4.6].

Here we address the case where the interaction between the $K$-trivial and the ML-random set is not having join above $\emptyset^{\prime}$. If $A$ is c.e. and not $K$-trivial, then $A \oplus Z=\emptyset^{\prime}$ for some ML-random $Z<_{T} \emptyset^{\prime}$ (see Theorem 2.2 below). We say that such a set $A$ is ML-cuppable. Thus, in analogy to Theorem 1.1, if a c.e. set $A$ is

Received by the editors June 17, 2005 and, in revised form, October 10, 2005.

2000 Mathematics Subject Classification. Primary 68Q30, 03D28.

Key words and phrases. Cupping, randomness, $K$-trivial.

The author was partially supported by the Marsden Fund of New Zealand, grant no. 03-UOA130.

(C)2006 American Mathematical Society Reverts to public domain 28 years from publication 
not ML-cuppable, then $A$ is $K$-trivial. (The hypotheses are quite different, though: in Theorem 1.1 the existence of an incomplete Martin-Löf-random set above $A$ is required, while here we require that no incomplete Martin-Löf- random set cups with $A$ to $\emptyset^{\prime}$.) How about existence of a non-computable $A$ in the non-cupping case? That is, is there a c.e. non-computable set which is not ML-cuppable? Our main result provides an affirmative answer. In fact one may choose $A$ promptly simple. We obtain the answer as a corollary to a more general non-cupping result: if $Y$ is $\Delta_{2}^{0}$ and ML-random, then there is a promptly simple set $A$ which does not help any ML-random set $Z$ to compute $A$, namely $Y \leq_{T} A \oplus Z \Rightarrow Y \leq_{T} Z$. Now let $Y=\Omega$ be Chaitin's halting probability, a Turing complete ML-random set. Note that each promptly simple set cups to $\emptyset^{\prime}$ by a low c.e. set [13, Thm XIII.4.2]. So $A$ is not ML-cuppable but cups to $\emptyset^{\prime}$ in the usual sense.

It is still an open question whether all $K$-trivial sets fail to be ML-cuppable [8, Question 4.8]. We will return to this question in the last section, after Corollary 3.5 .

\section{BACKGROUND}

The notion of ML-cuppability comes in two versions, depending on whether the cupping partner is merely not above $\emptyset^{\prime}$, or strictly below $\emptyset^{\prime}$.

Definition 2.1. A set $A$ is weakly $M L$-cuppable if $A \oplus Z \geq_{T} \emptyset^{\prime}$ for some ML-random set $Z \geq \emptyset^{\prime} . A$ is $M L$-cuppable if one can choose $Z<_{T} \emptyset^{\prime}$.

Kučera drew attention to Martin-Löf cuppability during his talk at a 2004 Cordoba meeting. In particular, he raised the question of which $\Delta_{2}^{0}$ sets are (weakly) ML-cuppable, and whether one of the notions is equivalent to $K$-triviality (see 8 , Question 4.8]). Quite a bit is known already. Let $\Omega^{A}$ be Chaitin's halting probability relativized to $A$ (see [1]). If the $\Delta_{2}^{0}$ set $A$ is not $K$-trivial, then $A$ is weakly ML-cuppable, via $Z=\Omega^{A}$. For $Z \geq_{T} \emptyset^{\prime}$ by Theorem 1.1, and $\emptyset^{\prime} \leq_{T} A^{\prime} \equiv_{T} A \oplus \Omega^{A}$. If $A$ is low, then in fact $\Omega^{A}<_{T} \emptyset^{\prime}$. Thus, each $\Delta_{2}^{0}$ set $A$ with a low non- $K$-trivial set below it is ML-cuppable. This includes:

a) any ML-random set $A$ since $A \cap 2 \mathbb{N}$ is low [1, Thm. 3.4],

b) any non-low ${ }_{2}$ and any c.e.a. non- $K$-trivial set, as each one of these is the supremum of a pair of 1-generic sets, and each 1-generic set is generalized low (see [7, Ex. IV.3.15] for the first),

c) any c.e. non- $K$-trivial set $A$, because $A$ is a disjoint union of c.e. low sets $A_{0}, A_{1}$, and at least one of them is not $K$-trivial [3].

By c) we have an analog of Theorem 1.1 .

Theorem 2.2. If a c.e. set $A$ is not $M L$-cuppable, then $A$ is $K$-trivial.

The question of whether each non- $K$-trivial $\Delta_{2}^{0}$ set is ML-cuppable is still not completely settled. An interesting case of a non- $K$-trivial $\Delta_{2}^{0}$ set which is not MLcuppable by any of the reasons above is a set of minimal degree which is properly $\mathrm{low}_{2}$ [7, Ex. IX.2.7].

Notation. We let $\mu$ denote the usual product measure in Cantor space $2^{\omega}$. The variable $\sigma$ denotes a string in $2^{<\omega}$. For a set $Z \in 2^{\omega}, Z\lceil n$ denotes $Z(0) \ldots Z(n-1)$. For $G \subseteq 2^{<\omega}$, we let $[G] \preceq$ denote the open set generated by $G$, that is, $\{Z: \exists \sigma \in$ $G \sigma \prec Z\}$. We write $\mu G$ for $\mu\left([G]^{\preceq}\right)$. 
For a set $A$ and a string $\sigma$, we let $A \oplus \sigma$ be the string $\rho$ of length $2|\sigma|$ such that $\rho(2 i)=A(i)$ and $\rho(2 i+1)=\sigma(i)$. Thus if $\Gamma$ is a Turing functional, then $\Gamma^{A \oplus \sigma}(x)$ is defined only when the use on the $A$-side is at most $|\sigma|$.

Given an expression $E$ that is approximated during stages $s, E[s]$ denotes its value at stage $s$.

A Solovay test is a c.e. set $\mathcal{S}$ of strings such that the weight $\sum_{\sigma \in \mathcal{S}} 2^{-|\sigma|}$ is finite. It is known that $Z$ is ML-random iff for each Solovay test $\mathcal{S}, \sigma \nprec Z$ for almost all $\sigma \in \mathcal{S}[2]$.

A c.e. set $A$ is promptly simple if $A$ is co-infinite and there is an effective approximation $\left(A_{s}\right)_{s \in \mathbb{N}}$ of $A$ such that, for each $e$, the requirement

$$
S_{e}:\left|W_{e}\right|=\infty \Rightarrow \exists s \exists x\left[x \in W_{e, s}-W_{e, s-1} \wedge x \in A_{s}\right]
$$

is met.

\section{MAIN RESUlt}

Our main theorem implies the existence of a non-computable c.e. set $A$ which is not ML-cuppable. By Theorem 2.2 such a set is $K$-trivial.

Theorem 3.1. Let $Y \in \Delta_{2}^{0}$ be Martin-Löf-random. Then there is a promptly simple set $A$ such that, for each Martin-Löf-random set $Z$,

$$
Y \leq_{T} A \oplus Z \Rightarrow Y \leq_{T} Z .
$$

Proof. We use [9, Lemma 4.2], in relativized form.

Lemma 3.2 (9]). Let $Y$ be $M L$-random relative to $A$. Then for each partial computable functional $\Gamma$, there is a constant $c$ such that for each $n$,

$$
\mu\left\{\sigma: Y\left\lceil n=\Gamma^{A \oplus \sigma}\lceil n\} \leq 2^{-n+c} .\right.\right.
$$

As the authors of 9 ] have pointed out, this is best proved via a supermartingale whose undergraph is c.e. in $A$. Let

$$
M(\eta)=2^{|\eta|} \mu\left\{\sigma: \Gamma^{A \oplus \sigma} \succeq \eta\right\} .
$$

Then, for each $\eta, M(\eta 0)+M(\eta 1) \leq 2 M(\eta)$, that is, $M$ is a supermartingale. Moreover, $\{\langle q, \eta\rangle: q \in \mathbb{Q} \wedge q \leq M(\eta)\}$ is c.e. relative to $A$. Since $Y$ is ML-random relative to $A$, there is $c$ such that $M(\eta) \leq 2^{c}$ for each $\eta$, which is (3.2).

Proof of Theorem 3.1, outline. Fix some effective approximation $\left(Y_{s}\right)_{s \in \mathbb{N}}$ of $Y$. To ensure that $Y$ is ML-random relative to $A$, we make $A K$-trivial; then $A$ is low for ML-random (namely each ML-random set is already ML-random relative to $A$ ) by [11. Thm 6.2]. To ensure that $A$ is promptly simple, we meet the requirements $S_{e}$ introduced above. Finally, we meet the non-cuppability requirements

$$
N_{\Gamma, c}: Y=\Gamma(A \oplus Z) \wedge c \text { is as in (3.2) } \wedge Z \text { ML-random } \Rightarrow Y \leq_{T} Z .
$$

Throughout, $N$ will denote some non-cuppability requirement. We define a $\Delta_{2}^{0}$ set $\Delta=\Delta_{N}$, which is an attempt to build a functional for $Y \leq_{T} Z$ by emulating $\Gamma$. Thus, when $Y \uparrow n=\Gamma^{A \oplus \sigma} \uparrow n$, we put the pair $\langle\sigma, Y\lceil n\rangle$ into $\Delta$. When $A \uparrow|\sigma|$ changes later, then this pair is removed from $\Delta$. At each stage, $\Delta$ has the monotonicity properties of a functional, namely $\sigma_{0} \preceq \sigma_{1} \wedge\left\langle\sigma_{i}, \eta_{i}\right\rangle \in \Delta \Rightarrow \eta_{0} \preceq \eta_{1}$. We also enumerate a Solovay test $\mathcal{S}=\mathcal{S}_{N}$ for each non-cuppability requirement $N$, which represents the "mistakes" we make due to $A$ changes: when a pair $\langle\sigma, y\rangle$ is removed from $\Delta$, then we put $\sigma$ into $\mathcal{S}$. If the hypothesis of $N$ is correct and we 
can ensure that $\mathcal{S}$ indeed is a Solovay test, then, since $Z$ is ML-random, $\sigma \notin \mathcal{S}$ for almost all $\sigma \prec Z$. Thus, eventually a pair $\langle\sigma, Y \uparrow n\rangle$ such that $\sigma \prec Z$ is not removed from $\Delta$, and we can pretend $\Delta$ is c.e. just like a Turing functional, which shows $Y \leq_{T} Z$.

Fix some effective priority ordering of the requirements of the form $N=N_{\Gamma, c}$ and of the prompt simplicity requirements $S_{e}$. To ensure $\mathcal{S}_{N}$ is a Solovay test, we have to minimize the number of $A$-changes. A requirement $S_{e}$ can at most add weight $2^{-e}$ to $\mathcal{S}_{N}$, for any requirement $N$ of higher priority. To give $S_{e}$ a chance, for each string $y$ of length $n$, at each stage $s$ we only allow a maximum measure of $2^{-n+c+2}$ for the set of $\sigma$ 's such that $\langle\sigma, y\rangle$ is in $\Delta_{N, s}$. Lemma 3.2 ensures that still we do not miss the right $\sigma$, namely the shortest $\sigma \prec Z$ such that $\Gamma^{A \oplus \sigma} \uparrow n=Y \uparrow n$.

Details. The construction consists in letting the requirements at stage $s$ carry out their strategies below, in the order of their priority, up to $s-1$.

Strategy for $N=N_{\Gamma, c}$. Let $\Delta_{N, 0}=\mathcal{S}_{N, 0}=\emptyset$. At stage $s>0$,

- (adding to $\Delta)$ For $n=0, \ldots, s-1$, do the following: while

$$
\mu\left\{\sigma:\left\langle\sigma, Y_{s-1} \mid n\right\rangle \in \Delta_{N, s-1}\right\} \leq 2^{-n+c+2},
$$

if there is $\sigma \notin \operatorname{dom}\left(\Delta_{N, s-1}\right)$ such that

$-\left\langle\sigma^{\prime}, Y_{s-1}\lceil n-1\rangle \in \Delta_{N, s-1}\right.$ for some $\sigma^{\prime} \preceq \sigma$ and

$-Y_{s-1} \uparrow n=\Gamma_{s-1}^{A_{s-1} \oplus \sigma} \uparrow n$,

then let $\sigma$ be such a string of minimal length and put $\left\langle\sigma, Y_{s-1}\lceil n\rangle\right.$ into $\Delta_{N}$.

- (removing from $\Delta$ ) If $\langle\sigma, y\rangle$ entered $\Delta$ at stage $t<s$ and $s$ is minimal such that $A_{s} \uparrow|\sigma| \neq A_{t} \uparrow|\sigma|$, then remove $\langle\sigma, y\rangle$ from $\Delta$, and put $\sigma$ into $\mathcal{S}_{N}$.

Strategy for $S_{e}$. Let $K_{t}(y)$ be the approximation of $K(y)$ at stage $t$, and let $\operatorname{cost}(x, t)=\sum_{x<y \leq t} 2^{-K_{t}(y)}$ be the usual cost function from [3] (also see [11]). For each $N=N_{\Gamma, c}$, let

$$
\operatorname{cost}_{N}(x, t)=\mu\left\{\sigma: \exists y\langle\sigma, y\rangle \in \Delta_{N, t-1} \wedge x<|\sigma|\right\}
$$

be the cost, in the sense of increasing the weight of $N$ 's Solovay test $\mathcal{S}_{N}$, one would incur by putting $x$ into $A$ at stage $t$. At stage $s$, suppose $S_{e}$ is not satisfied and there is $x \in W_{e, s}-W_{e, s-1}, x \geq 2 e$, such that $\operatorname{cost}(x, s) \leq 2^{-e-1}$ and $\operatorname{cost}_{N}(x, s) \leq 2^{-e}$ for each requirement of type $N$ of higher priority. If $x$ is not in $A_{s}$ yet, then put $x$ into $A_{s}$. Declare $S_{e}$ satisfied.

Verifications. $A$ is $K$-trivial by the usual argument involving the cost function (see [3] or [11, Prop 4.1]). Also $\mathcal{S}_{N}$ is a Solovay test for each requirement $N$. To see this, note that each requirement $S_{e}$ acts at most once. Thus the requirements of stronger priority than $N$ contribute a finite weight, and each requirement $S_{e}$ of lower priority than $N$ contributes at most weight $2^{-e}$ to $\mathcal{S}_{N}$, so their total is at most 2 .

Lemma 3.3. A is promptly simple.

Proof. Clearly $A$ is co-infinite. Thus we only need to verify that each requirement $S_{e}$ is met. It suffices to show that for each requirement of type $N$, for sufficiently large $x$,

$$
\forall s \geq x \operatorname{cost}_{N}(x, s) \leq 2^{-e}
$$


For in that case, if $x \geq 2 e$ enters $W_{e}$ at stage $s$, then $x$ can be enumerated into $A$ in case $x$ is so large that $\operatorname{cost}(x, s) \leq 2^{-e-1}$ and, for all $N$ type requirements of stronger priority than $S_{e}, \operatorname{cost}_{N}(x, s) \leq 2^{-e}$.

To show that (3.4) holds for sufficiently large $x$, we split the cost into two parts, depending on whether a computation $\Gamma^{A \oplus \sigma}(k)[s]$ for $k<e+c+3$ would be destroyed by the enumeration of $x$ into $A$ or not.

Bounding the cost of the first type. Fix $i$, and let $r \in \mathbb{N}$ be given. We show that, for sufficiently large $x$, the cost incurred by enumerating $x$ into $A$ and thereby destroying a computation $\Gamma^{A \oplus \sigma}(k), k<i$ (that is, removing a pair $\langle\sigma, y\rangle$ from $\Delta_{N}$ where $|y|=i)$, can be kept below $2^{-r+1}$. We later will apply this to all $i \leq e+c+3$, for $r=e+i+2$, so that the contribution is at most $2^{-i-e-1}$ for sufficiently large $x$. The argument is similar to the one of Kučera and Terwijn [6] in their construction of a non-computable low for ML-random set. Consider the open $A$-c.e. set $B_{i}^{A}=\left\{\sigma: \Gamma^{A \oplus \sigma} \uparrow i \downarrow\right\}$, with the usual approximation $B_{i}^{A}[t]=\left\{\sigma: \Gamma_{t}^{A_{t} \oplus \sigma}\lceil i \downarrow\}\right.$, and let

$$
\operatorname{cost}_{i}(x, s)=\mu\left\{\sigma \in B_{i}^{A}[s-1]: x<|\sigma|\right\} .
$$

(By the convention in Section 2, $x<|\sigma|$ implies $x<$ use $\Gamma_{s}^{A_{s} \oplus \sigma} \uparrow i$. Note that there is no dependence here between the costs for different $i$ : for smaller $i$ we have more pairs $\langle\sigma, y\rangle$ in $\Delta$ where $|y|=i$ but the use on $A$ is smaller; for larger $i$ there are fewer pairs but the use is larger.) We claim that, for each $r$, there is $x$ such that

$$
\forall s \geq x \operatorname{cost}_{i}(x, s) \leq 2^{-r+1} .
$$

To show this, recall that each $S_{k}$ acts at most once. So if no $S_{k}, k \leq r$, acts from stage $t_{0}$ on, then for each $t \geq t_{0}$, for each $s \geq t, \mu B_{i}^{A}[s] \geq \mu B_{i}^{A}[t]-2^{-r}$. That is, $\mu B_{i}^{A}[t]$ can only decrease by $2^{-r}$ after stage $t_{0}$. Thus, for each $t \geq t_{0}$, $\mu B_{i}^{A}[t] \leq \mu B_{i}^{A}+2^{-r}$. Now choose $x \geq t_{0}$ so that $\mu B_{i}^{A}[x] \geq \mu B_{i}^{A}-2^{-r}$, with $A_{x}$ correct on the use of the relevant computations $\Gamma^{A \oplus \sigma}(k), k<i$. Then for all $s \geq x$, $\operatorname{cost}_{i}(x, s) \leq 2^{-r+1}$.

Bounding the cost of the second type. Let $s_{0}$ be a stage so that $\widetilde{y}=Y_{s} \uparrow e+c+3$ is stable from $s_{0}$ on. By the usual conventions, enumeration of $x \geq s_{0}$ into $A$ cannot destroy any computation $\Gamma^{A \oplus \sigma}(k)$ existing at stage $s_{0}$. So if $x \geq s_{0}$, we only have to consider the contribution of pairs $\langle\sigma, y\rangle$ to the cost of the second type (i.e., where $|y| \geq e+c+3$ ) which entered $\Delta$ after stage $s_{0}$. In this case $\widetilde{y} \preceq y$. For $s \geq s_{0}$, let

$$
F_{s}=\left\{\rho:\langle\rho, \widetilde{y}\rangle \in \Delta_{s}\right\} .
$$

Then $F_{s}$ is an antichain and, by (3.3),$\mu F_{s} \leq 2^{-(e+c+3)+c+2}=2^{-e-1}$.

Now consider the situation where $x \geq s_{0}$ and a pair $\langle\sigma, y\rangle$ entered $\Delta$ at stage $u \geq s_{0}$ and is in $\Delta_{t}$ for all $t, u \leq t \leq s$. If $|\sigma|>x$, then this pair is counted in the calculation of $\operatorname{cost}_{N}(x, s)$. If $|y|>e+c+3$, then by choice of $s_{0}, \widetilde{y} \preceq y$. In the construction, we ensured that $\langle\rho, \widetilde{y}\rangle \in \Delta_{u}$ for some $\rho \preceq \sigma$. Then $\langle\rho, \widetilde{y}\rangle \in \Delta_{s}$ since $A_{u} \uparrow|\rho|=A_{s} \uparrow|\rho|$, and hence $\sigma \in\left[F_{s}\right] \preceq$. So one can bound the cost of the second type by $\mu F_{s}$.

Summarizing the two separate arguments, we obtain (3.4): for $x$ sufficiently large, for all $N$ of higher priority than $S_{e}, \operatorname{cost}_{N}(x, s) \leq \sum_{i \leq e+c+3} 2^{-i-e-1}+$ $2^{-e-1} \leq 2^{-e}$

Lemma 3.4. If $Y=\Gamma(A \oplus Z)$ and $Z$ is $M L$-random, then $Y \leq_{T} Z$.

Proof. Since $A$ is $K$-trivial, $Y$ is ML-random relative to $A$ [11, Thm 7.2]. Let $c$ be as in Lemma 3.2. and let $N=N_{\Gamma, c}$. First we make two observations. 
(a) For each $p$, a pair $\left\langle\sigma, Y\lceil p\rangle\right.$ enters $\Delta_{N}$, where $\sigma \prec Z$.

To see this, informally speaking, we have to show that the measure condition (3.3) is not too restrictive. Choose $t$ such that $Y_{s} \uparrow p=Y_{t} \uparrow p$ for each $s \geq t$, and no requirement $S_{k}$ acts after $t$ for $k \leq p$. For any $n \leq p$, by Lemma 3.2,

$$
\mu\left\{\sigma: \Gamma^{A \oplus \sigma}\lceil n=Y \uparrow n\} \leq 2^{-n+c} .\right.
$$

Then at any stage $s \geq t, \mu\left\{\sigma:\left\langle\sigma, Y\lceil n\rangle \in \Delta_{N, s}\right\} \leq 2^{-n+c}+2^{-n} \leq 2^{-n+c+1}\right.$, because a requirement $S_{k}, k>p$, has to ensure that $\operatorname{cost}_{N}(x, s) \leq 2^{-k}$ and hence $\mu\left\{\sigma:\left\langle\sigma, Y\lceil n\rangle \in \Delta_{N, s}\right\}\right.$ can decrease at most $2^{-n}$ after stage $s$. So by the first stage $s \geq t$ where $\Gamma_{s}^{A_{s} \oplus Z} \uparrow p=Y \uparrow p$, for all $n=0, \ldots, p$ we may put a pair $\left\langle\sigma, Y\lceil n\rangle\right.$ where $\sigma \prec Z$ into $\Delta_{N}$ if it is not there already.

(b) Since $\mathcal{S}_{N}$ is a Solovay test and $Z$ is $M L$-random, there is $s_{0}$ such that if $\langle\sigma, y\rangle$ enters $\Delta_{N}$ at a stage $s \geq s_{0}$ where $\sigma \prec Z$, then $\sigma \notin \mathcal{S}_{N}$, and hence $\langle\sigma, y\rangle$ remains in $\Delta_{N}$ forever. Thus $A_{s} \uparrow|\sigma|$ is stable and hence $y \prec Y$.

We now give a procedure with oracle $Z$ which for almost all inputs $m$ returns $Y(m)$. Wait for a stage $s$ such that $\langle\sigma, y\rangle$ enters $\Delta_{N}$ at $s$, where $\sigma \prec Z$ and $|y|>m$. Then output $y(m)$. By (a) the procedure terminates, and by (b) the output is correct for almost all $m$, namely, whenever $s \geq s_{0}$. Thus $Y \leq_{T} Z$. The theorem is proved.

Corollary 3.5. There is a (necessarily $K$-trivial) promptly simple set $A$ which is not weakly ML-cuppable.

Proof. Let $Y$ be Chaitin's halting probability $\Omega$. Since $Y \equiv_{T} \emptyset^{\prime}$, the result follows from Theorem 3.1

Discussion. Let us compare our construction of a promptly simple set $A$ in the proof of Theorem 3.1 with the usual cost function construction of a promptly simple $K$-trivial set ([3] or [11, Prop 4.1]). Typically, a cost functions $c(x, s)$ restricts a prompt simplicity requirement $S_{e}$ by stipulating that at stage $s, x$ can enter $A$ for the sake of $S_{e}$ only if $c(x, s) \leq 2^{-e}$. First consider the usual cost function for achieving $K$-triviality, $\operatorname{cost}(x, s)=\sum_{x<y \leq s} 2^{-K_{s}(y)}$. Then for $x>s, \operatorname{cost}(x, s)=0$. If at a later stage $t>s$, we have $c(x, t)>2^{-e}$, then we may as well assume that the entire interval $[x, t)$ becomes unusable for $S_{e}$ (as the numbers with short descriptions at stage $t$ might be close to $t$ ). So $S_{e}$ will have to look for future candidates among the numbers $\geq t$. Now, for the usual cost function this process of intervals becoming unusable can repeat at most $2^{e}$ times, as it corresponds to an increase in the measure of the domain of the universal machine by $2^{-e}$. A cost functions $\operatorname{cost}_{N}$ has a similar restraining behavior on $S_{e}$, but now the process of intervals becoming unusable can repeat arbitrarily often. To see this, recall how we bounded the cost of the second type in the proof of Lemma 3.3. When $Y \uparrow e+c+3$ changes another time at $t$, then we have to restrict $S_{e}$ to numbers $\geq t$ in order to make the argument work. This is similar to Kučera's construction of a promptly simple set $A$ below a ML-random $\Delta_{2}^{0}$ set $Z$. Here, when $Z \uparrow e$ changes at stage $t$, then all numbers $<t$ become unusable for $S_{e}$ (see [10, Chapter 3]).

By [11, Thm 7.3], the $K$-trivial sets are precisely the ones that can be obtained via a cost function construction in terms of the usual function $\operatorname{cost}(x, s)$. Our discussion gives some evidence that the sets obtained by Kučera's construction (Theorem 1.1 and the remark after) form a proper subclass of the $K$-trivial sets. However, for the non-cupping construction, if $Y=\Omega$, then the process of restricting 
$S_{e}$ to larger intervals can only repeat $2^{e+c+3}$ times. So there is no clear evidence if the non-ML-cuppable sets form a proper subclass of the $K$-trivial sets.

To what extent does $A$ in Theorem 3.1 depend on $Y$ ? At this stage we do not even know whether there is a non-computable (c.e.) set $A$ such that (3.1) holds for all ML-random $Y, Z$. The construction in the proof of the Main Theorem can be modified in order to obtain $A$ which satisfies (3.1) for all ML-random $Y, Z$ such that $Y \leq_{\mathrm{wtt}} \emptyset^{\prime}$ (that is, $Y$ is $\omega$-c.e.).

Corollary 3.6. There is a promptly simple set $A$ such that for all $M L$-random sets $Y \leq_{\mathrm{wtt}} \emptyset^{\prime}$ and $Z$,

$$
Y \leq_{T} A \oplus Z \Rightarrow Y \leq_{T} Z
$$

Proof. We sketch the necessary modifications. Let $\left(\Psi_{i}\right)_{i \in \mathbb{N}}$ be an effective listing of wtt-reduction procedures and $Y_{i}=\Psi_{i}\left(\emptyset^{\prime}\right)$. We have an approximation $Y_{i, s}(x) \simeq$ $\Psi_{i}\left(\emptyset^{\prime}\right)[s]$, which may have the value 'undefined', but can change its value only finitely often. We now satisfy requirements $N=N_{\Gamma, c, i}$ which refer to $Y_{i}$ instead of $Y$. The strategy is as before, except that $N$ can only put a pair $\left\langle\sigma, Y_{i, s}\lceil n\rangle\right.$ into $\Delta$ at stage $s$ when $Y_{i, s}(k)$ is defined for all $k<n$. In the proof of Lemma 3.3 (which did not use that $Y$ is ML-random), there still is a stage $s_{0}$ from which on $Y_{i, s} \uparrow e+c+3$ is stable. If some value $Y_{i, s_{0}}(k)$ is undefined for $k<e+c+3$, then there is no contribution to the cost of the second type. Else we may argue as before.

\section{ADDED IN PROOF}

After this paper was submitted, Hirschfeldt gave an alternative, simpler proof of Corollary 3.5. See the last section of [12.

\section{ACKNOWLEDGMENTS}

Special thanks go to Sandra Moguel for her support. This paper is dedicated to her. I thank Antonin Kučera, Yu Liang, and in particular Joseph Miller for helpful discussions.

\section{REFERENCES}

1. R. Downey, D. Hirschfeldt, J. Miller, and A. Nies. Relativizing Chaitin's halting probability. To appear in J. Math. Logic.

2. Rod G. Downey and Denis R. Hirschfeldt. Algorithmic randomness and complexity. SpringerVerlag, Berlin. To appear.

3. Rod G. Downey, Denis R. Hirschfeldt, André Nies, and Frank Stephan. Trivial reals. In Proceedings of the 7th and 8th Asian Logic Conferences, pages 103-131, Singapore, 2003. Singapore Univ. Press. MR2051976 (2005a:03089)

4. D. Hirschfeldt, A. Nies, and F. Stephan. Using random sets as oracles. To appear.

5. A. Kučera. Measure, $\Pi_{1}^{0}$-classes and complete extensions of PA. In Recursion theory week (Oberwolfach, 1984), volume 1141 of Lecture Notes in Math., pages 245-259. Springer, Berlin, 1985. MR.0820784 (87e:03102)

6. A. Kucera and S. Terwijn. Lowness for the class of random sets. J. Symbolic Logic, 64:13961402, 1999. MR1780059 (2001j:03082)

7. M. Lerman. Degrees of Unsolvability. Perspectives in Mathematical Logic. Springer-Verlag, Heidelberg, 1983. MR0708718 (85h:03044)

8. J. Miller and A. Nies. Randomness and computability: open questions. To appear.

9. Joseph S. Miller and Liang Yu. On initial segment complexity and degrees of randomness. To appear in Trans. Amer. Math. Soc.

10. A. Nies. Computability and Randomness. Monograph, to appear. 
11. A. Nies. Lowness properties and randomness. Adv. in Math., 197:274-305, 2005. MR2166184

12. A. Nies. Eliminating concepts. To appear in Proceedings of IMS Workshop on Computational Prospects of Infinity, Singapore, 2005.

13. R. Soare. Recursively Enumerable Sets and Degrees. Perspectives in Mathematical Logic, Omega Series. Springer-Verlag, Heidelberg, 1987. MR0882921 (88m:03003)

Department of Computer Science, Private Bag 92019, Auckland, New Zealand

E-mail address: andre@cs.auckland.ac.nz 